University of Nebraska - Lincoln

DigitalCommons@University of Nebraska - Lincoln

Timothy J. Gay Publications

Research Papers in Physics and Astronomy

November 2006

\title{
Fluorescence polarization of helium negative-ion resonances excited by polarized electron impact
}

J. W. Maseberg

University of Nebraska - Lincoln

Timothy J. Gay

University of Nebraska - Lincoln, tgay1@unl.edu

Follow this and additional works at: https://digitalcommons.unl.edu/physicsgay

Part of the Physics Commons

Maseberg, J. W. and Gay, Timothy J. , "Fluorescence polarization of helium negative-ion resonances excited by polarized electron impact" (2006). Timothy J. Gay Publications. 46.

https://digitalcommons.unl.edu/physicsgay/46

This Article is brought to you for free and open access by the Research Papers in Physics and Astronomy at DigitalCommons@University of Nebraska - Lincoln. It has been accepted for inclusion in Timothy J. Gay Publications by an authorized administrator of DigitalCommons@University of Nebraska - Lincoln. 
Published in Journal of Physics B: Atomic, Molecular and Optical Physics 39 (2006) 4861-4870; doi:10.1088/0953-4075/39/23/005 Copyright (C) 2006 IOP Publishing Ltd. Used by permission. Online at http://stacks.iop.org/JPhysB/39/4861

Submitted September 13, 2006; revised October 4, 2006; published November 10, 2006.

\title{
Fluorescence polarization of helium negative-ion resonances excited by polarized electron impact
}

\author{
J. W. Maseberg and T. J. Gay \\ Behlen Laboratory of Physics, University of Nebraska-Lincoln, \\ Lincoln, NE 68588-0111, USA; email: maseberg@bigred.unl.edu
}

\begin{abstract}
We have investigated helium $(1 \mathrm{~s} 3 \mathrm{~d}){ }^{3} \mathrm{D} \rightarrow(1 \mathrm{~s} 2 \mathrm{p}){ }^{3} \mathrm{P}(588 \mathrm{~nm})$ fluorescence produced by electron impact excitation in the vicinity of the $\left(2 \mathrm{~s}^{2} 2 \mathrm{p}\right)^{2} \mathrm{P}$ and $\left(2 \mathrm{~s} 2 \mathrm{p}^{2}\right)$ ${ }^{2} \mathrm{D}$ negative-ion resonances at 57.2 and $58.3 \mathrm{eV}$, respectively. In contrast to previous work, we use spin-polarized incident electrons and report the relative Stokes parameters $P_{1}, P_{2}$, and $P_{3}$ in the $55-60 \mathrm{eV}$ region. Our failure to see discernable resonance effects in $P_{2}$ indicates that even though the lifetime of these resonances is significant ( $\sim 10 \mathrm{fs})$, magnetic forces acting on the temporarily captured electron are small. Resonant structures in the values of $P_{1}$ and $P_{3}$ are observed because the polarization contributions of resonant states are generally different than those from direct excitation of the $3{ }^{3} \mathrm{D}$ state.
\end{abstract}

\section{Introduction}

Helium resonance structures located above the ionization potential were first discovered in a transmission experiment by Kuyatt et al. [1]. Two features having energies near 57.2 and $58.3 \mathrm{eV}$ were then tentatively classified as $\mathrm{He}^{-}\left(2 \mathrm{~s}^{2} 2 \mathrm{p}\right){ }^{2} \mathrm{P}$ and $\left(2 \mathrm{~s}^{2} \mathrm{p}^{2}\right){ }^{2} \mathrm{D}$ states by Fano and Cooper [2]. These designations have since been confirmed [3, 4]. An accurate measurement of the resonance energies performed by Hicks et al. [5] found values of 57.22(4) and 58.30(4) eV, respectively, which are in excellent agreement with other experimental and theoretical results $[6,7]$.

Numerous researchers have done electron transmission and energy-loss experiments to characterize these and other helium negative-ion resonances [8]. Another method of investigation consists of monitoring the fluorescence of states that result from decay of these negative-ion resonances. In particular, the intensity and linear polarization of the $3{ }^{3} \mathrm{D} \rightarrow$ $2{ }^{3} \mathrm{P}$ transition have been studied following excitation with unpolarized incident electron beams [9-14]. (The resonance features in this transition are particularly pronounced.) The work we present here is similar, but uses incident spin-polarized electrons. The process of interest is 


$$
\begin{aligned}
\mathrm{e}+\mathrm{He}\left(1 \mathrm{~s}^{2}\right)^{1} \mathrm{~S} & \rightarrow \mathrm{He}^{-}\left(2 \mathrm{~s}^{2} 2 \mathrm{p}\right){ }^{2} \mathrm{P} \text { or } \mathrm{He}^{-}\left(2 \mathrm{~s} 2 \mathrm{p}^{2}\right)^{2} \mathrm{D} \\
& \rightarrow \mathrm{He}(1 \mathrm{~s} 3 \mathrm{~d}){ }^{3} \mathrm{D}+\mathrm{e} \\
& \rightarrow \mathrm{He}(1 \mathrm{~s} 2 \mathrm{p}){ }^{3} \mathrm{P}+\mathrm{e}+\gamma(588 \mathrm{~nm}) .
\end{aligned}
$$

Interference between these channels and the direct excitation of the $3{ }^{3} \mathrm{D}$ state produces resonant features in the observed intensity well characterized by Beutler-Fano profiles $[15,16]$. Cascade contributions from the $4 p, 5 p$ and $6 p$ levels are responsible for $\sim 50 \%$ of the observed $588 \mathrm{~nm}$ radiation in the $50-60 \mathrm{eV}$ energy range [13]. However, the effects of the resonances on the emitted radiation from the $4 p$ and $5 p$ states are known to be small [10], and we expect the same for the $6 p$ state. Therefore, subsequent cascades from these states to the $3{ }^{3} \mathrm{D}$ level do little to affect the resonance features we discuss below and will be ignored in the following discussion.

The polarization of the emitted photons is described by Stokes parameters and has been shown to be sensitive to exchange effects and magnetic forces [17]. The Stokes parameters are defined in the standard way to be

$$
I=s_{0}, \quad P_{1}=s_{1} / s_{0}, \quad P_{2}=s_{2} / s_{0} \quad \text { and } \quad P_{3}=s_{3} / s_{0},
$$

where $s_{0}, s_{1}, s_{2}$, and $s_{3}$ are the components of the Stokes vector. The parameter $I$ represents the intensity of emitted photons. The $P_{1}$ linear polarization represents the intensity asymmetry for electric field vectors aligned parallel and perpendicular to the electron beam axis. Similarly, $P_{2}$ is the linear polarization given by the intensity asymmetry for electric field vectors aligned parallel and perpendicular to an axis which is rotated by $45^{\circ}$ from the beam. The circular polarization parameter $P_{3}$ corresponds to an intensity asymmetry for right-handed versus left-handed helicity.

When the electron beam is transversely spin-polarized and the scattered electrons are not detected, Stokes parameters $P_{2}$ and $P_{3}$ are not required to be zero as they are in the unpolarized case [18]. The motivation for this experiment was to investigate these values carefully as a function of incident electron energy near the resonances. Features observed in these regions could be a signature of relativistic magnetic forces acting during the resonance lifetime. While such forces are generally small in light atoms, the resonance lifetime ( $\sim 10 \mathrm{fs}$ ), roughly 50 times longer than the classical orbital period for $n=2$ states of $\mathrm{He}$, could reasonably be expected to enhance their influence. If electron spin precession occurred in the triply excited resonance due to magnetic forces, measurement of a non-zero value of $P_{2}$ and variations in $P_{3}$ would be allowed because spin could no longer be factored out of the interaction Hamiltonian. Equivalently, one could say that the compound ion state was not well- $L \mathrm{~S}$ coupled. In the case of $P_{2}$, discernable structures near the resonance energies would be a clear indication that magnetic forces are present. Resonance structures in $P_{3}$ can be caused by two processes, the first being the magnetic interactions discussed above. Alternatively, variations in $P_{3}$ might occur because Coulombic interactions are generally different for the interfering resonance and direct channels leading to $3{ }^{3} \mathrm{D}$ formation. Since $P_{3}$ depends on the initial distribution of ML states (which affect $P_{1}$ even more directly [17]), any difference between the resonant and direct ML distributions will yield a variation in $P_{3}$ across the resonance profile.

\section{Experiment}

Details of our apparatus have been previously presented [19-22], but a brief overview is given here. The apparatus is shown in figure 1. To obtain polarized electrons, an un- 


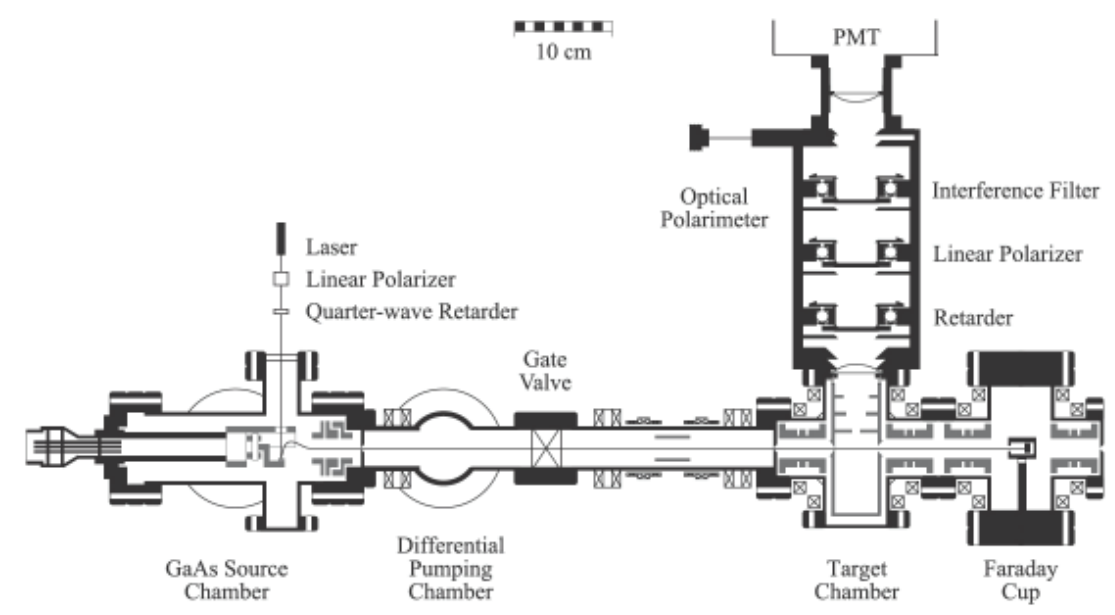

Figure 1. Experimental apparatus.

strained bulk GaAs photocathode is chemically etched and placed in the source chamber. After bakeout, a base pressure of $1 \times 10^{-8} \mathrm{~Pa}$ is reached. The crystal is then resistively heat-cleaned and activated with caesium and oxygen to produce typical photocurrents of $5 \mu \mathrm{A} \mathrm{mW}^{-1}$ with incident $785 \mathrm{~nm}$ laser light. The helicity of the laser light determines the spin of the photoemitted electrons and is controlled by rotating a quarter-wave retarder. The electrons are electrostatically deflected by $90^{\circ}$ to produce a beam with transverse polarization and steered into a $5 \mathrm{~cm}$ long target gas cell. Helium pressure in the cell was kept at $8 \times 10^{-2} \mathrm{~Pa}$ to minimize the effects of radiation trapping [13]. Electrons pass through the target cell and are collected in a Faraday cup. The collision energy is defined by the target cell voltage. Differential pumping regions isolate the source pressure from that of the target. The target cell is topped with a $50 \mathrm{~mm}$ plano-convex vacuum lens, upon which is mounted an optical polarimeter. Three optical stages consisting of a linear retarder, linear polarizer and interference filter are followed by another plano-convex lens that focuses light onto a cooled GaAs photomultiplier tube for photon counting.

\section{Data analysis}

Data are acquired by rotating the polarimeter retarder and measuring count rates at $22.5^{\circ}$ intervals with the linear polarizer pass-axis fixed parallel to the electron beam. This method is advantageous as it allows for simultaneous measurement of all Stokes parameters. It also eliminates the potential problem of polarization-sensitive detection, as the linear polarizer remains fixed in place after initial alignment. For each position of the retarder, the collision energy is varied and photons are counted. When all preselected energies have been scanned the retarder is advanced to a new position. After one full rotation of the retarder the source quarter-wave retarder is advanced by $90^{\circ}$, changing the sign of the electron beam polarization. Background counts are subtracted from the raw photon signal, which is then normalized to beam current and target pressure.

For this configuration, the measured relative intensity $I^{\prime}$ can be related to the Stokes parameters describing the initial optical state using Mueller matrices. A rotatable retarder of retardance $\delta$ with its fast axis located at an angle $\beta+\beta_{0}$ acting on the initial state, fol- 
lowed by a linear polarizer positioned at an angle $\alpha_{0}$, yields

$$
\begin{aligned}
I_{i}^{\prime} \propto I\left\{1+\frac{1}{2}\right. & P_{1} k_{\mathrm{inc}}\left\lceil\cos \left(2 \alpha_{0}\right)\left[\cos \left(4 \beta_{i}+4 \beta_{0}\right)(1-\cos \delta)+(1+\cos \delta)\right]\right. \\
& \left.+\sin \left(2 \alpha_{0}\right) \sin \left(4 \beta_{i}+4 \beta_{0}\right)(1-\cos \delta)\right\rfloor \\
& +\frac{1}{2} P_{2} k_{\mathrm{inc}}\left[\cos \left(2 \alpha_{0}\right) \sin \left(4 \beta_{i}+4 \beta_{0}\right)(1-\cos \delta)\right. \\
& \left.-\sin \left(2 \alpha_{0}\right)\left[\cos \left(4 \beta_{i}+4 \beta_{0}\right)(1-\cos \delta)-(1+\cos \delta)\right]\right\rfloor \\
& \left.-P_{3} k_{\mathrm{inc}} \sin \left(2 \beta_{i}+2 \beta_{0}-2 \alpha_{0}\right) \sin \delta\right\},
\end{aligned}
$$

where $\alpha_{0}, \beta_{0}, \delta$, and $k_{\text {inc }}$ are constants. Since $\alpha_{0}$ and $\beta_{0}$ are the offset angles for the linear polarizer and linear retarder, respectively, they are ideally zero. Their adjustability serves to correct for physical misalignments. The retardance is optimally $90^{\circ}$ for the wavelength of interest, but in practice it must be independently measured. The kinc parameter must also be measured and is defined as $k_{\text {inc }}=\left(k_{1}-k_{2}\right) /\left(k_{1}+k_{2}\right)$, where $k_{1}$ and $k_{2}$ are the maximum and minimum transmittances of completely linearly polarized light. The retarder angle $\beta_{\mathrm{i}}=(i-1) \times 22.5^{\circ}$, where $i=1,2, \ldots, 16$. Extracting expressions for Stokes parameters are accomplished by multiplying (3) with appropriate sinusoidal terms and summing over one full revolution of retarder positions. This gives

$$
\begin{aligned}
& I \propto I^{\prime \prime}=f_{0}-2(1+\cos \delta)\left[f_{1} \cos \left(4 \alpha_{0}\right)+f_{2} \sin \left(4 \alpha_{0}\right)\right] /(1-\cos \delta), \\
& P_{1}=4\left[f_{1} \cos \left(2 \alpha_{0}\right)+f_{2} \sin \left(2 \alpha_{0}\right)\right] /\left[I^{\prime \prime} k_{\text {inc }}(1-\cos \delta)\right], \\
& P_{2}=4\left[f_{2} \cos \left(2 \alpha_{0}\right)-f_{1} \sin \left(2 \alpha_{0}\right)\right] /\left[I^{\prime \prime} k_{\text {inc }}(1-\cos \delta)\right] \text { and } \\
& P_{3}=-2 f_{3} /\left(I^{\prime \prime} k_{\text {inc }} \sin \delta\right),
\end{aligned}
$$

where $f_{0}, f_{1}, f_{2}$, and $f_{3}$ are defined as

$$
\begin{aligned}
& f_{0} \equiv \frac{1}{16} \sum_{i=1}^{16} I_{i}^{\prime}, \\
& f_{1} \equiv \frac{1}{16} \sum_{i=1}^{16} I_{i}^{\prime} \cos \left(4 \beta_{i}+4 \beta_{0}\right) \\
& f_{2} \equiv \frac{1}{16} \sum_{i=1}^{16} I_{i}^{\prime} \sin \left(4 \beta_{i}+4 \beta_{0}\right) \quad \text { and } \\
& f_{3} \equiv \frac{1}{16} \sum_{i=1}^{16} I_{i}^{\prime} \sin \left(2 \beta_{i}+2 \beta_{0}-2 \alpha_{0}\right) .
\end{aligned}
$$

Each rotation of the retarder results in one set of Stokes parameter measurements for each energy. Final values are obtained by averaging multiple measurements together; the standard deviation of the mean of these distributions is used to determine uncertainties. We applied Chauvenet's criterion to the data to eliminate occasional grossly erroneous count rates from the photomultiplier tube. The parameters $\alpha_{0}$ and $\beta_{0}$ are varied and chosen in such a way that the global weighted mean of all $P_{1}$ values is maximized and the spin-unnormalized weighted mean of $P_{2}$ is made zero. The spin-unnormalized value $P_{3}$ is then also found to be zero within its statistical uncertainty.

To determine the electron beam polarization, the Stokes parameters for the $389 \mathrm{~nm}$ helium $(1 \mathrm{~s} 3 \mathrm{p}) 3{ }^{3} \mathrm{P} \rightarrow(1 \mathrm{~s} 2 \mathrm{~s}) 2{ }^{3} \mathrm{~S}$ transition were measured [23, 24]. The beam polarization is given by

$$
P_{\mathrm{e}}=\frac{6 P_{3}}{3-P_{1}}
$$




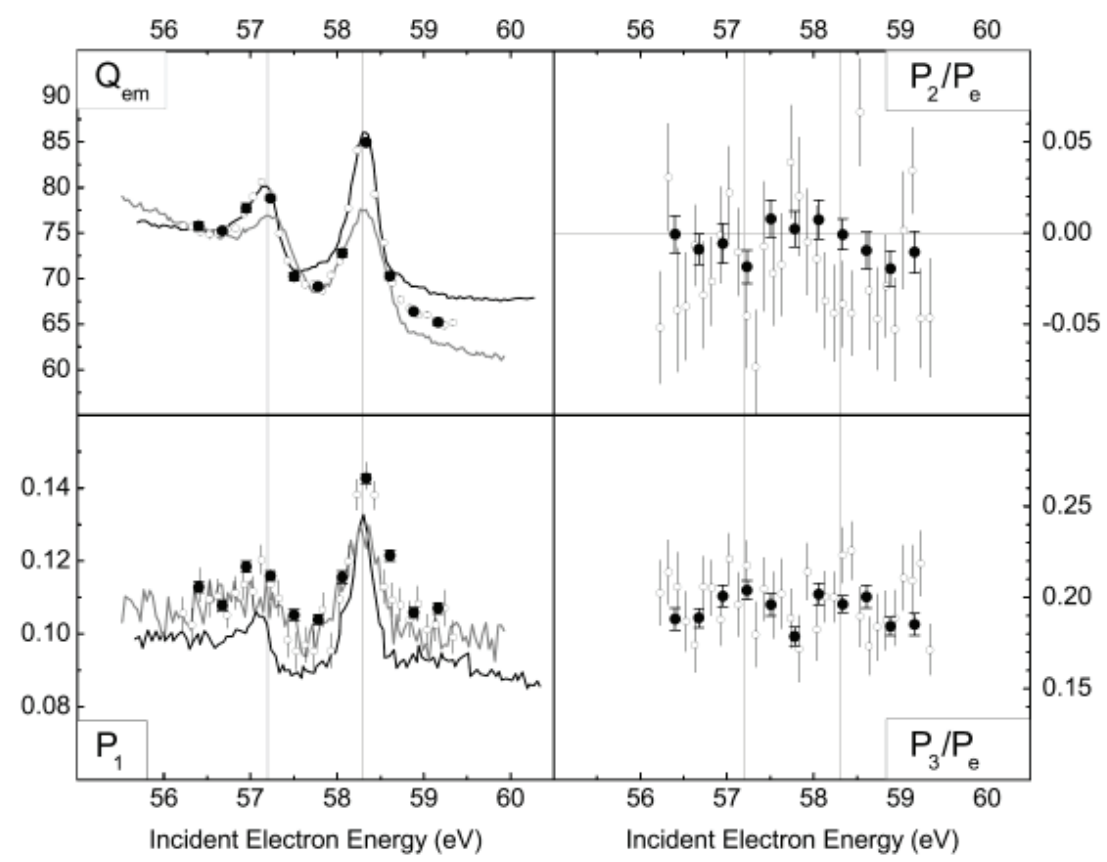

Figure 2. Relative emission cross section $Q_{\mathrm{em}}$ (in arbitrary units) and Stokes parameters $P_{1}, P_{2} / P_{\mathrm{e}}$ and $P_{3} / P_{\mathrm{e}}$. Our data are shown as empty and filled circles (two separate experimental runs), the values of Defrance [10] are displayed as black lines and the work of Cvejanović et al. $[13,14]$ is represented with gray lines. Resonance energies of 57.2 and $58.3 \mathrm{eV}$ are indicated.

For the data presented in this paper we measured a beam polarization of $P_{\mathrm{e}}=0.144(4)$. This value is unusually low; normally, we measure beam polarizations between 0.20 and 0.30 . We attribute this discrepancy to the fact that the crystal had been resistively heatcleaned many times prior to acquisition of the present data.

\section{Results and discussion}

\section{Intensity and relative emission}

The Stokes parameter $I$ is a quantity that represents the photon intensity emitted into a small solid angle ( 0.12 steradians in our case). It is not directly proportional to a relative emission cross section, as it is dependent on the angular distribution of the atomic radiation. If photons are collected perpendicular to the electron beam direction, the polarization-independent relative emission cross section $Q_{\mathrm{em}}$ is given by [25]

$$
Q_{\mathrm{em}} \propto I\left(1-P_{1} / 3\right)
$$

Results from this experiment have not been corrected for the finite optical collection angle or electron beam divergence because the magnitude of these corrections is within the statistical uncertainty of the data. The intensity, relative emission cross section and linear polarization fraction $P_{1}$ are all independent of the polarization of the incident electron beam, and thus can be directly compared to work done with unpolarized electrons. The graph in figure 2 shows the relative emission cross section for the $\mathrm{He} 3{ }^{3} \mathrm{D} \rightarrow 2{ }^{3} \mathrm{P}$ transition. The values on the $y$-scale are representative of our experimental collection rate in $\mathrm{Hz}$. The data sets of other references were normalized to our lowest energy point. 


\section{Linear polarization $P_{1}$}

The observed intensity near the resonances can be described by the convolution of an apparatus profile with a modified Beutler-Fano function of the form

$$
I^{\|, \perp}=I_{\mathrm{b}}^{\|, \perp}+I_{1}^{\|, \perp}\left[\frac{\left(q_{1}^{\|, \perp}+\epsilon_{1}\right)^{2}}{1+\left(\epsilon_{1}\right)^{2}}\right]+I_{2}^{\|, \perp}\left[\frac{\left(q_{2}^{\|, \perp}+\epsilon_{2}\right)^{2}}{1+\left(\epsilon_{2}\right)^{2}}\right],
$$

where $\epsilon_{r}=2\left(E-E_{r}\right) / \Gamma_{r}$ and $\Gamma_{r}$ is the FWHM of a given resonance with energy $E_{r}$. Here, the $r$ indices 1 and 2 refer to the ${ }^{2} \mathrm{P}$ and ${ }^{2} \mathrm{D}$ peaks, respectively. The dimensionless shape parameter is given by $q_{r}$, and the superscripts indicate the collection of light with linear polarization oriented either parallel or perpendicular to the incident electron beam. The background far from the resonances is then

$$
B^{\|, \perp}=I_{\mathrm{b}}^{\|, \perp}+I_{1}^{\|, \perp}+I_{2}^{\|, \perp},
$$

where $I_{\mathrm{b}}$ is treated as a function of energy while $I_{1}$ and $I_{2}$ are taken as constants. The "resonance polarization" (with $\epsilon_{1}=0$ for the ${ }^{2} \mathrm{P}$ resonance and $\epsilon_{2}=0$ for the ${ }^{2} \mathrm{D}$ resonance) is then defined by Defrance [10] to be

$$
P_{r}\left(\epsilon_{1} \text { or } \epsilon_{2}=0\right)=\frac{\left[I^{1}-B^{\|}\right]-\left[I^{\perp}-B^{\perp}\right]}{\left[I^{\|}-B^{\|}\right]+\left[I^{\perp}-B^{\perp}\right]} .
$$

As pointed out by Batelaan et al. [12], this definition of the resonance polarization depends not only on parameters associated with the resonant process, but also on the direct excitation cross section. Batelaan et al. [12] have shown that the light intensity for a given $3{ }^{3} \mathrm{DM}_{\mathrm{L}}$ state can be expressed as

$$
I=I^{\mathrm{dir}}+I^{\mathrm{res}}-I_{\mathrm{sym}}^{\mathrm{int}}+I_{\mathrm{asym}}^{\mathrm{int}},
$$

where $I^{\mathrm{dir}}$ and $I^{\text {res }}$ can be identified uniquely with the direct excitation and resonant excitation processes, and $I_{\mathrm{sym}}^{\mathrm{int}}$ and $I_{\mathrm{asym}}^{\mathrm{int}}$ correspond to symmetric and antisymmetric interference terms. Because $I^{\text {res }}$ and $I$ int sym exhibit the same energy dependence, they are not distinguishable in the type of experiment discussed in this paper. Equation (9) implicitly contains both these terms, and as such cannot be formally identified as the "resonance polarization," i.e., the polarization associated with a purely resonant process.

This being said, we have evaluated $\mathrm{P}_{r}$ for the sake of comparison with earlier work. We not only analyze our data, but also that of Defrance [10] and Cvejanović et al. [13, 14]. The data of Batelaan et al. [12] is excluded because we could not obtain it. Resonance polarizations are calculated by fitting data of a given polarization state with a convolution of (7) with an apparatus profile describing the electron beam energy characteristics. In order to approximate the electron beam shape profile, the sum of normalized triangle $(\mathrm{T})$ and normalized Lorentzian (L) functions (both with identical widths) is used so that the profile is expressed as $w T(E)+(1-w) L(E)$, where the weighting factor $w$ obeys $0 \leq w \leq 1$. The convolution of this apparatus profile with (7) gives a tractable analytical expression [26], and the triangle term closely represents the numerical result obtained when using a Gaussian instead. This form is somewhat arbitrary, but it gives reasonable results and, lacking detailed knowledge of the beam profiles, seems justifiable. A quadratic form is chosen for the background dependence $I_{\mathrm{b}}$. The resonance widths (0.071 and $\left.0.047 \mathrm{eV}[4]\right)$ and energy separation (1.094 eV [6]) are held fixed to facilitate convergence. Fitting is done with a standard Levenberg-Marquardt algorithm, and after convergence is obtained all parameters are fixed (assuming zero uncertainty in the fitting parameters) except for the $I_{1}$ and $I_{2}$ amplitudes. The subsequent error estimates for these values are used to determine the uncertainty in the resonance polarization. 


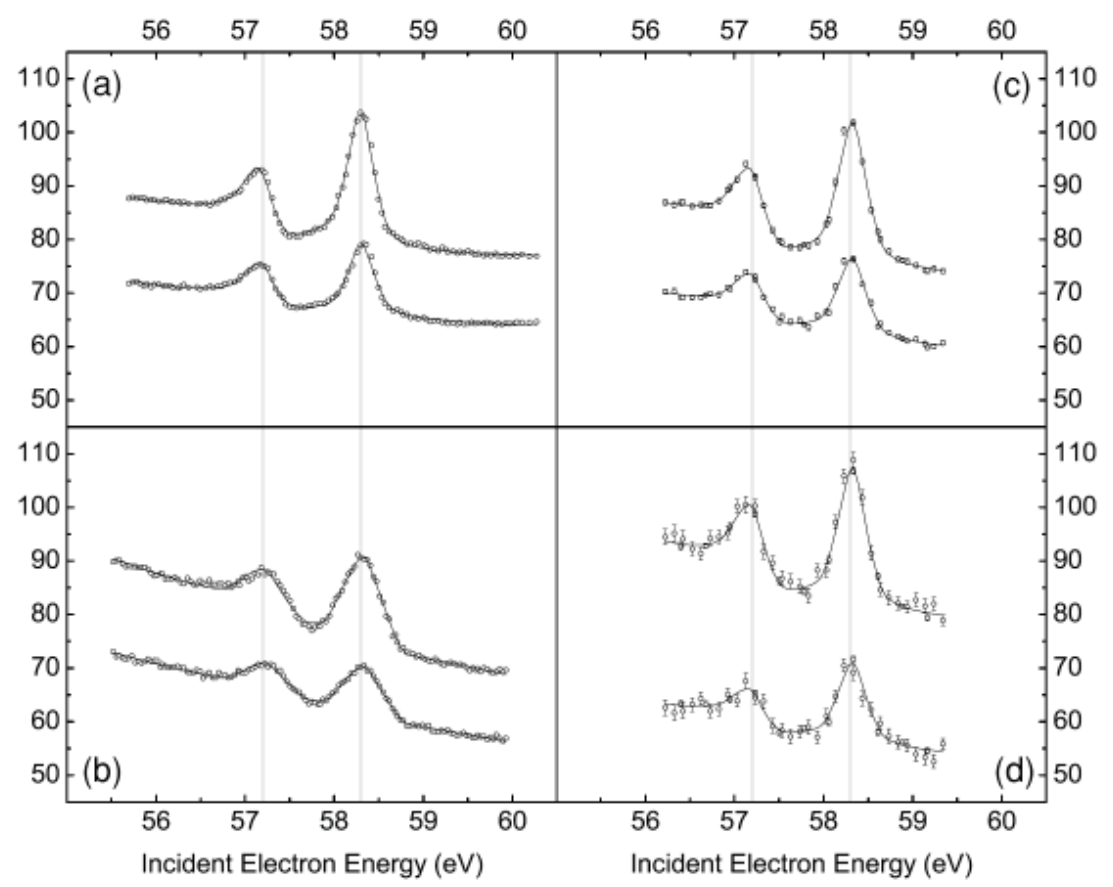

Figure 3. Graphs (a)-(c) contain parallel and perpendicularly polarized intensities (in arbitrary units) from Defrance [10], Cvejanović et al. [13, 14] and this work, respectively. Graph (d) contains our right-and left-handed polarized intensities normalized to electron spin. Fits to the data are represented as solid black lines. Resonance energies of 57.2 and $58.3 \mathrm{eV}$ are indicated.

The fits are shown in graphs (a)-(c) of figure 3. The energy widths of the electron beams in the different experiments are thus estimated by us to be $0.29,0.43$, and $0.33 \mathrm{eV}$, and the weighting factors $\mathrm{w}$ are approximately $0.3,1.0$, and 0.4 for the data in (a), (b) and (c), respectively. The resulting $\mathrm{q}^{\|, \perp}$ values for the ${ }^{2} \mathrm{P}$ state are in the range of -2.6 to -11 , and for the ${ }^{2} \mathrm{D}$ case lie between -11 and -42 for the fits. These are similar to results reported by Defrance and de Froment [9]. The reduced $\chi^{2}$ values for all fits in figure 3 lie between 0.14 and 1.2. Previously reported resonant polarizations and the results from our fits are shown in table 1.

Comparison of the previously reported results for $P_{r}$ (upper part of table 1) shows that Cvejanović et al. [13] and Batelaan et al. [12] are in close agreement, while Defrance's values are about $3 \sigma$ away for the ${ }^{2} \mathrm{P}$ resonance and less than $2 \sigma$ away for the ${ }^{2} \mathrm{D}$ state. This seems to indicate that the results of Defrance are in error. By using the same values of the resonance widths and separation energy in our fits to all the available data, the situation changes somewhat (lower part of table 1). Our results for the ${ }^{2} \mathrm{P}$ resonance indicate that previous work is in good agreement and that our value is about $2 \sigma$ larger, while for the ${ }^{2} \mathrm{D}$ resonance all polarizations are in reasonable agreement.

Our data support the interesting conclusion that $P_{r}$ for both resonances is nearly consistent with the kinematically demanded value for non-interfering resonant state production, followed by decay to the $\mathrm{He}(1 \mathrm{~s} 3 \mathrm{~d}){ }^{3} \mathrm{D}$ state and an outgoing electron, with the outgoing electron in its lowest allowed angular momentum partial wave $[10,12]$. In the case of the ${ }^{2} \mathrm{P}$ resonance, this is an $l=1$ wave, and $P_{r}$ is expected to be 0.24 as computed by van Ittersum (see [12] and [27]). For the ${ }^{2} \mathrm{D}$ resonance, the outgoing electron can have $l$ 
Table 1. Linear polarization fractions for the resonances as defined by equation (9).

\begin{tabular}{|c|c|c|c|c|}
\hline State & Present work & Cvejanović et al [13] & Batelaan et al [12] & Defrance [10] \\
\hline \multicolumn{5}{|c|}{ Previously reported values } \\
\hline${ }^{2} \mathrm{P}(57.2 \mathrm{eV})$ & - & $0.13 \pm 0.03$ & $0.14 \pm 0.02$ & $0.24 \pm 0.03$ \\
\hline${ }^{2} \mathrm{D}(58.3 \mathrm{eV})$ & - & $0.247 \pm 0.001$ & $0.25 \pm 0.02$ & $0.30 \pm 0.03$ \\
\hline \multicolumn{5}{|c|}{ Our results and results we obtained by fitting the data of others } \\
\hline${ }^{2} \mathrm{P}(57.2 \mathrm{eV})$ & $0.22 \pm 0.03$ & $0.16 \pm 0.02$ & - & $0.13 \pm 0.02$ \\
\hline${ }^{2} \mathrm{D}(58.3 \mathrm{eV})$ & $0.29 \pm 0.01$ & $0.27 \pm 0.01$ & - & $0.30 \pm 0.01$ \\
\hline
\end{tabular}

$=0$ and for this case $P_{r}$ should be 0.32 [27] (this is the same as that required for threshold polarization of the $3{ }^{3} \mathrm{D}$ state). This is remarkable because, as mentioned earlier, the use of (9) does not allow measurement of the pure resonant polarization. Thus, we conclude, as did Batelaan et al. [12], that the symmetric interference contribution $\underset{\mathrm{sym}}{\underset{\mathrm{int}}{\mathrm{y}} \text { is either }}$ small or exhibits a similar $\mathrm{M}_{\mathrm{L}}$ dependence as the resonant $\mathrm{I}^{\text {res }}$ term (which leads to equivalent light polarizations). Higher order allowed outgoing partial waves could also contribute to some extent, which may account for the fact that our measurements are slightly lower than the required threshold values. No further conclusions can be drawn from the present experiment, but we note that the definitive measurement of resonant polarizations must involve some mechanism to distinguish between I ${ }^{\text {res }}$ and I ${ }_{\text {sym }}^{\text {int }}$. The fitting procedure to extract resonant polarizations would also benefit from increased electron beam energy resolution.

\section{Linear polarization $P_{2} / P_{\mathrm{e}}$}

Our data for $P_{2} / P_{\mathrm{e}}$ shown in figure 2 is comprised of two different experimental runs. The first run (open circles) contains more energy steps and greater statistical uncertainty. The weighted mean computed using all energies is $-0.019(5)$ for the first data set and 0.006 (3) for the second. Even though the $3{ }^{3} \mathrm{D}$ state is well- $L \mathrm{~S}$ coupled, it is possible that cascading from higher lying non-well- $L S$ coupled states could produce non-zero values of $P_{2}$ [21]. If this were the case, one would expect the marginally non-zero $P_{2}$ values observed to be essentially independent of energy over the 55-60 eV range, given that resonant cascading is expected to be small (as mentioned in the introduction). We are quite certain that the non-zero measured $P_{2}$ values are not due to stray magnetic fields or optical misalignments, as we have chosen the offset angles in (4) such that $P_{2}$ is zero for an unpolarized electron beam. However, we have no explanation for the statistical inconsistency between the two data sets. Interestingly, the open-circle data fail a Shapiro-Wilk normality test at the 0.05 significance level, meaning they do not obey Gaussian statistics. Therefore, we cannot be confident that our non-zero values of $P_{2}$ are indicative of cascading from higher lying non-well- $L S$ coupled states, as the data sets are inconsistent with each other.

The question of whether $P_{2} / P_{\mathrm{e}}$ reveals structures at the resonant energies is of greater importance. Resonant $P_{2}$ polarizations can be extracted using a similar procedure as that described for $P_{1}$ polarizations. This gives $-0.22(9)$ and $-0.04(3)$ for the ${ }^{2} \mathrm{P}$ and ${ }^{2} \mathrm{D}$ resonances, respectively. If there were no magnetic interactions, one would expect zero for both resonant polarizations; for the ${ }^{2} \mathrm{P}$ state the extracted value is $2.5 \sigma$ away from zero. However, the filled circle data point at the ${ }^{2} \mathrm{P}$ resonance energy in figure 2 is $2 \sigma$ off the zero line (which is consistent with the above analysis), but due to the similar scatter of other data points, we do not attribute this to a feature. The reduced $\chi^{2}$ of a linear fit (with zero slope) to the filled circle data points is 0.90 , which argues against the presence of any statistically significant structure. 


\section{Circular polarization $P_{3} / P_{\mathrm{e}}$}

Figure 2 shows $P_{3} / P$ e, and there appears to be some structure at the resonance peaks. We attribute these features to Coulombic (as opposed to magnetic) interactions. There are two reasons for this. First, the lack of any obvious resonance structure in the $P_{2}$ data indicates that resonant magnetic effects are negligible, as discussed in the introduction. Secondly, the features we observe are consistent with a resonant cascade-free value of $P_{3}$. In the absence of resonant processes, the direct excitation of the $3{ }^{3} \mathrm{D}$ state via electron exchange produces a kinematically required threshold polarization of 0.25 . As cascading becomes more important at higher energies $(55-60 \mathrm{eV}), P_{3}$ decreases and we measure it to be $\sim 0.19$ (figure 2). The reduced $\chi^{2}$ value from a linear fit to the filled circle $P_{3} / P$ e data is 2.6, strengthening our assertion that the structures are indeed real.

Using the same techniques as those used for calculating $P_{r}$, we determine that the "resonance circular polarizations" for the ${ }^{2} \mathrm{P}$ and ${ }^{2} \mathrm{D}$ features are $0.37(6)$ and $0.26(2)$, respectively. The fits are shown in graph (d) of figure 3. Since these are within $2 \sigma$ of the threshold value of 0.25 , we argue simply that resonant processes give a value of $P_{3}$ in agreement with cascade-free exchange excitation. This is not surprising, given that no variation in $P_{2}$ (i.e., magnetic precession of electron spin in the transient resonant state) is observed.

\section{Conclusion}

Having found no statistically significant structure for Stokes parameter $P_{2}$ in the helium $3{ }^{3} \mathrm{D} \rightarrow 2{ }^{3} \mathrm{P}$ transition, we conclude that magnetic spin-orbit interactions in the negative-ion $\left(2 s^{2} 2 p\right){ }^{2} \mathrm{P}$ and $\left(2 \mathrm{~s} 2 \mathrm{p}^{2}\right)^{2} \mathrm{D}$ resonant states are not important and present an upper bound of $P_{2} / P_{\mathrm{e}} \leq 0.02$ for this effect. Measured values for the linear "resonant polarization" fractions as defined by Defrance are consistent with the kinematically required threshold values for $3{ }^{3} \mathrm{D} \rightarrow 2{ }^{3} \mathrm{P}$ radiation, assuming the outgoing electron is in the lowest allowed angular momentum state. Our values for the circular "resonant polarization" are in fair agreement with the threshold value required by exchange excitation of the 3 ${ }^{3} \mathrm{D}$ state, with the caveat that the computed resonance polarizations are not solely due to polarization from resonance state decays, but in principle can be coupled to polarization from the direct excitation of the $3{ }^{3} \mathrm{D}$ state through interference phenomena.

\section{Acknowledgments}

The authors thank Herman Batelaan for useful discussions. This work was supported by the NSF under grant PHY-0354946.

\section{References}

[1] Kuyatt C E, Simpson J A, and Mielczarek S R 1965 Phys. Rev. 138 A385-99

[2] Fano U and Cooper J W 1965 Phys. Rev. 138 A400-2

[3] Trantham K W, Jacka M, Rau A R P, and Buckman S J 1999 J. Phys. B: At. Mol. Opt. Phys. 32 $815-24$

[4] Nicolaides C A and Piangos N A 2001 Phys. Rev. A 64 052505-17

[5] Hicks P J, Cvejanović S, Comer J, Read J M, and Sharp J M 1974 Vacuum 24 573-80

[6] Roy D 1977 Phys. Rev. Lett. 38 1062-65

[7] Bylicki M and Nicolaides C A 1995 Phys. Rev. A 51 204-10

[8] Buckman S J and Clark C W 1994 Rev. Mod. Phys. 66 539-655 
[9] Defrance A and de Froment G 1977 Proc. 10th Int. Conf. on Physics of Electronic and Atomic Collisions vol. 2 pp 692-3

[10] Defrance A 1980 J. Phys. B: At. Mol. Phys. 13 1229-48

[11] van der Burgt P J M, van Eck J, and Heideman H G M 1986 J. Phys. B: At. Mol. Phys. 19 2015-37

[12] Batelaan H, van Eck J, and Heideman H G M 1991 J. Phys. B: At. Mol. Opt. Phys. 24 5151-67

[13] Cvejanović D, Clague K, Fursa D, Bartschat K, Bray I, and Crowe A 2000 J. Phys. B: At. Mol. Opt. Phys. 33 2265-78

[14] Crowe A 2006 personal communication (corrected data)

[15] Fano U and Cooper J W 1965 Phys. Rev. 137 A1364-79

[16] Fano U 1961 Phys. Rev. 124 1866-78

[17] Furst J E, Wijayaratna W M K P, Madison D H, and Gay T J 1993 Phys. Rev. A 47 3775-87

[18] Bartschat K and Blum K 1982 Z. Phys. A 304 85-8

[19] Al-Khateeb H M, Birdsey B G, and Gay T J 2005 Phys. Rev. A 71 032707-15

[20] Al-Khateeb H M, Birdsey B G, and Gay T J 2000 Phys. Rev. Lett. 85 4040-3

[21] Birdsey B G, Al-Khateeb H M, Johnston M E, Bowen T C, Gay T J, Zeman V, and Bartschat K 1999 Phys. Rev. A 60 1046-52

[22] Al-Khateeb H M, Birdsey B G, Bowen T C, Green A S, Johnston M E, and Gay T J 1999 Rev. Sci. Instrum. $703882-5$

[23] Gay T J, Furst J E, Trantham K W, and Wijayaratna W M K P 1996 Phys. Rev. A 53 1623-29

[24] Gay T J 1983 J. Phys. B: At. Mol. Phys. 16 L553-56

[25] Clout P N and Heddle D W O 1969 J. Opt. Soc. Am. 59 715-7

[26] Jimenez-Mier J 1994 J. Quantum Spectrosc. Radiat. Transfer 51 741-9

[27] Heddle D W O, Keesing R G W, and Parkin A 1977 Proc. R. Soc. A 352 419-28 